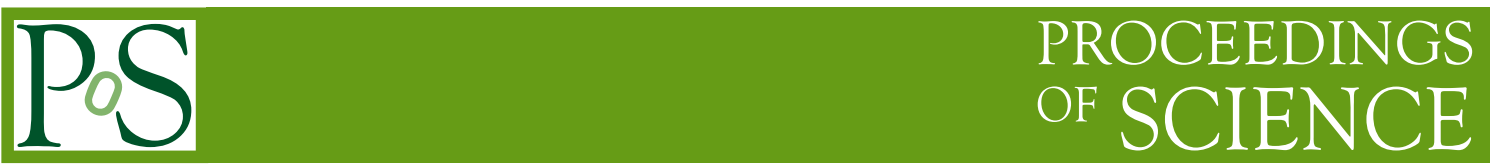

\title{
The COMPASS experiment at CERN
}

\author{
Oleg Kouznetsov ${ }^{* \dagger}$ \\ JINR, Dubna \\ E-mail: Oleg.Kouznetsovecern.ch
}

COMPASS (COmmon Muon and Proton Apparatus for Structure and Spectroscopy) is a fixed target experiment at CERN dedicated to studies of the spin structure of the nucleon and of the spectroscopy of hadrons. During the years 2002-2004, 2006-2007 and 2010 the COMPASS collaboration has collected a large amount of data by scattering polarized $160 \mathrm{GeV} / \mathrm{c}$ muons on polarized ${ }^{6} \mathrm{LiD}$ and $\mathrm{NH}_{3}$ targets. These data were used in particulary to evaluate the gluon contribution $\Delta G$ to the nucleon spin, to measure the helicity quark distributions $x \Delta q(x)$ and for study the transversity effects.

In 2008-2009 COMPASS performed a search for $\mathrm{J}^{\mathrm{PC}}$-exotic mesons, glueballs or hybrids, through light hadron spectroscopy in high energy $190 \mathrm{GeV} / \mathrm{c} \pi^{-}$-proton reactions using both centrally produced and diffractive events. Preliminary results from these searches are discussed.

Finally the future COMPASS-II experimental program beyond 2011 is presented. A study of Generalised Parton Distributions (GPDs) will be done in exclusive reactions like Deeply Virtual Compton Scattering (DVCS) and Deeply Virtual Meson Production (DVMP). In parallel the study of flavour separation and hadron fragmentation in Semi-Inclusive Deep Inelastic Scattering (SIDIS) will be continued. Drell-Yan (DY) processes will be used for study of Transverse Momentum Dependent distributions (TMDs) using a transversely polarized target. A Primakoff reaction will be used to extract pion and kaon polarisabilities.

The XXth International Workshop High Energy Physics and Quantum Field Theory

September 24-October 1, 2011

Sochi Russia

\footnotetext{
*Speaker.

${ }^{\dagger}$ on behalf of the COMPASS Collaboration
} 


\section{THE COMPASS EXPERIMENT}

The goal of the COMPASS experiment at CERN is to scrutinize how nucleons and other hadrons are built up from quarks and gluons. The main physics observables studied by the Collaboration are the polarization of the constituents of a polarized nucleon, the mass and decay patterns of the light hadronic system with either exotic quantum numbers or strong gluonic excitation. At hard scales Quantum Chromodynamics (QCD) is well established but in the non-perturbative regime, despite the numerous experimental data, a fundamental understanding of hadronic structure is still missing.

COMPASS takes advantage of a variety of high energy and high intensity beams (muons and hadrons) being located at the M2 beam line of CERN's Super Proton Synchrotron (SPS). The COMPASS set-up was designed for beams of 100 to $200 \mathrm{GeV} / \mathrm{c}$ and was built around two large dipole magnets, defining two consecutive spectrometers, covering large and small scattering angles separately Fig.11. To match the expected particle flux in the various locations along the spectrom-

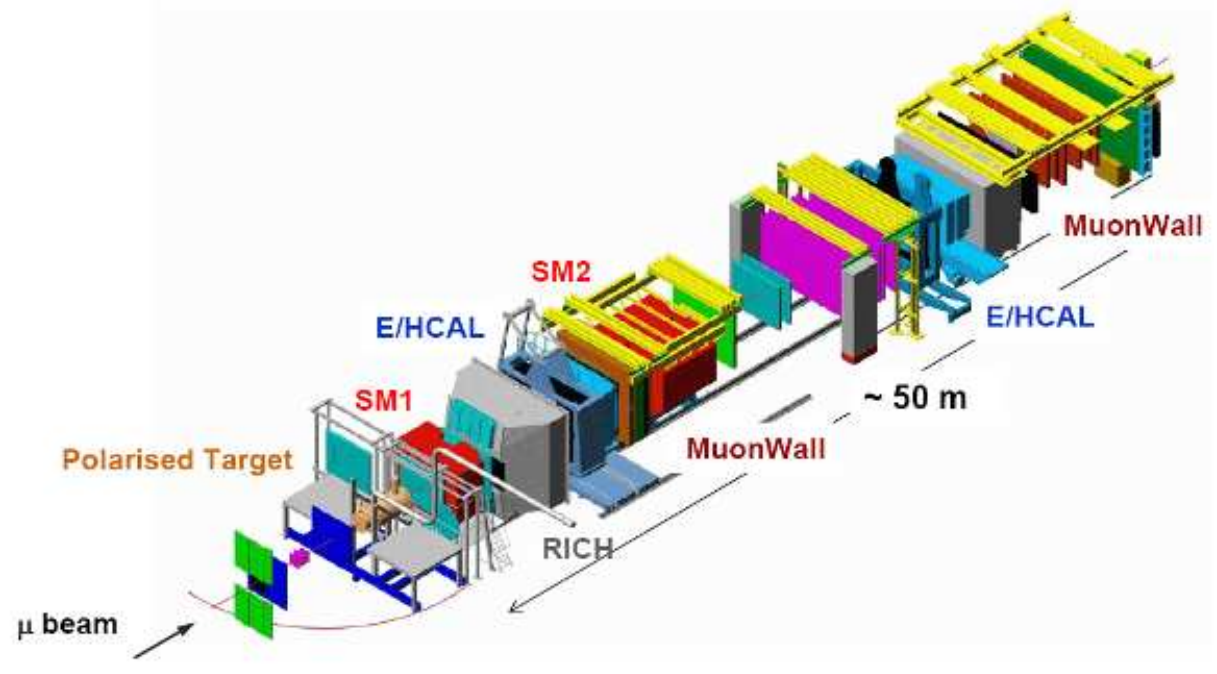

Figure 1: Layout of the COMPASS spectrometer.

eter, COMPASS uses wide variety of very different tracking detectors: silicon detectors and scintillating fibers, Micromegas and GEM micromesh detectors, large proportional and drift chambers. Particle identification is performed using a RICH counter and both electromagnetic and hadron calorimeters. The polarized target (actually the largest polarized target in the world), consists of two oppositely polarized cells, $60 \mathrm{~cm}$ long each, surrounded by a large solenoid superconducting magnet. Until 2006, the two cells were filled with a ${ }^{6} \mathrm{LiD}$ target material (mainly deuterium), for which polarizations higher than 50\% were routinely achieved. In 2007, 2010-2011 the ammonia $\left(\mathrm{NH}_{3}\right.$, mainly proton) target, reaching polarizations of $90 \%$ and higher was used. Also, the target material has been distributed in three cells, of 30,60 and $30 \mathrm{~cm}$ length. Since 2006 a new target magnet has been installed, increasing the acceptance from $\pm 70 \mathrm{mrad}$ to $\pm 180 \mathrm{mrad}$. A full description of the spectrometer can be found in [1].

This article consists of three parts. The first part is dedicated to the study of the nucleon spin structure (2003-2007 and 2010 years). The second part presents the results from the hadron reac- 
tions (2004 short test run and 2008-2009 years). In the third part the COMPASS-II experimental program for next 3 years starting from 2012 will be discussed.

\section{Part I: NUCLEON SPIN STRUCTURE}

\subsection{A "spin-crisis" and the gluon polarization measurements}

Since 1988, when the EMC experiment found that only a small fraction of the nucleon spin is carried by the quarks, $\Delta \Sigma=12 \pm 9 \pm 14 \%$ [2], the origin of the spin of the nucleon remains an intriguing puzzle. The discrepancy between this measurement and an expectation following from the relativistic quark models, which predict that $60 \%$ of the nucleon spin should come from the spin of quark and anti-quark constituents [3], was named the "spin-crisis". The EMC result has been confirmed by series of deep inelastic scattering experiments at CERN, SLAC and DESY, giving, on average, a contribution from the quarks $\Delta \Sigma$ to the nucleon spin of $\sim 30 \%$.

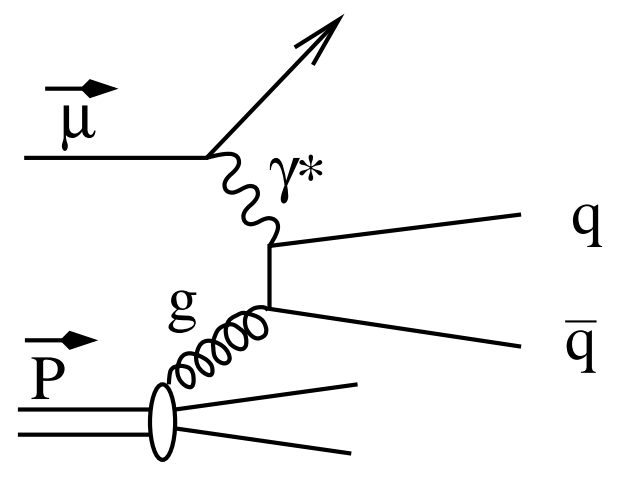

Figure 2: The photon gluon fusion process, used for direct measurements of the gluon polarization. Fragmentation of the created $q \bar{q}$ pairs into charmed D mesons gives a sample of events with minimal background for a $\frac{\Delta g}{g}$ measurement.
The spin $1 / 2$ of the nucleon can be decomposed as $1 / 2=1 / 2 \Delta \Sigma+\Delta G+L_{q+g}$ and one concludes that the missing contribution to the nucleon spin must come from the gluons $\Delta G$, and/or from the orbital angular momenta $L_{q+g}$. The measurement of the gluon polarization is very important since a possible solution of the "spin-crisis" and violation of the Ellis-Jaffe sum rule [ [ $]$ is produced if $\Delta G$ is of order of 3 . Here $\Delta G$ is the first moment of the gluon helicity distribution $\Delta g\left(x_{g}\right)$. Experimentally, the polarization $\frac{\Delta g\left(x_{g}\right)}{g\left(x_{g}\right)} 1$ of gluons carrying a fraction $x_{g}$ of nucleon momentum is measured.

The gluon polarization can be directly measured via the spin asymmetry of the Photon-Gluon Fusion (PGF) process, shown in Fig.2. In this process the fusion of the virtual photon and the gluon creates a quark-antiquark pair in the final state. The fragmenting $q \bar{q}$ pairs are then detected with two different, but complementary methods.

a) The "open charm" [ [ 7 method. The cleanest way to tag the PGF process is the $\gamma^{*} g \rightarrow c \bar{c}$ chain because the presence of $c$ quarks inside the nucleon is negligibly small. The created $c \bar{c}$ pairs predominantly fragment into vector and pseudoscalar charmed-non-strange mesons. The constraint on mass difference $\Delta M=M_{D^{*}}-M_{D^{0}}$ (so-called " $\mathrm{D}^{*}$ tag") was used for the effective combinatorial background suppression. In total $90600 \mathrm{D}^{0}$ mesons were reconstructed [ $₫$ ] in the following decay $\mathrm{D}^{0} \rightarrow \mathrm{K} \pi, \mathrm{D}^{0} \rightarrow \mathrm{K} \pi \pi^{0}$ and $\mathrm{D}^{0} \rightarrow \mathrm{K} \pi \pi$ modes.

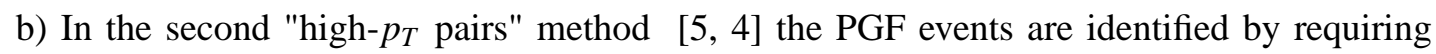
that two opposite-charge high-transverse momentum hadrons are detected in coincidence. In the "high- $p_{T}$ pairs" analysis the spin helicity asymmetry is calculated by selecting events containing hadrons with $p_{T}$ above 0.7 and $0.4 \mathrm{GeV} / \mathrm{c}$, for the first and for the second hadron with respect the

\footnotetext{
${ }^{1}$ Further instead of $\frac{\Delta g\left(x_{g}\right)}{g\left(x_{g}\right)}$ a simplified notation as $\frac{\Delta g}{g}$ will be used for gluon polarization non averaged on $x_{g}$ interval and $\left\langle\frac{\Delta g}{g}\right\rangle$ for the averaged one.
} 
virtual photon direction. Since this method selects light quarks as well, its counting rate is high; so the competing background processes play an important role introducing model dependence of its description. On the contrary the "open charm" leptoproduction method is clean, free from physics background but statistically limited.

All the gluon polarization measurements of the COMPASS are summarized in Fig.; together with the SMC [6] and HERMES [7] results. It should be stressed that the worlds result for direct measurements of the $\left\langle\frac{\Delta g}{g}\right\rangle$ is dominated by COMPASS and indicates a small value of $\Delta G$. Similarly, the global QCD analysis [8] of $g_{1}$ data also indicates a small value of the first moment of $\Delta g:|\Delta G|$ $=0.2-0.3$. To solve the "spin crisis" 10 times bigger $|\Delta G|$ is needed. These results are the probable signature for a predominant role of the angular orbital momentum of quarks and gluons in nucleon spin decomposition.
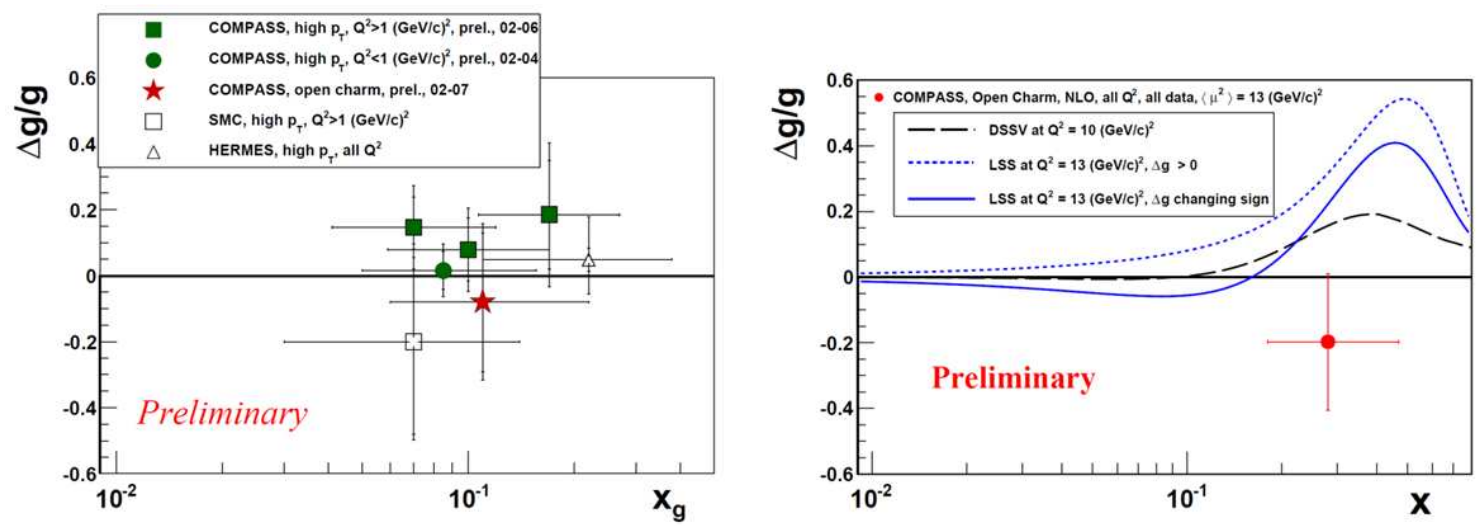

Figure 3: Summary of the world efforts for the direct $\left\langle\frac{\Delta g}{g}\right\rangle$ measurement: LO QCD order results Left, NLO QCD order results Right.

\subsection{The helicity quark distriburions and the transversity effects.}

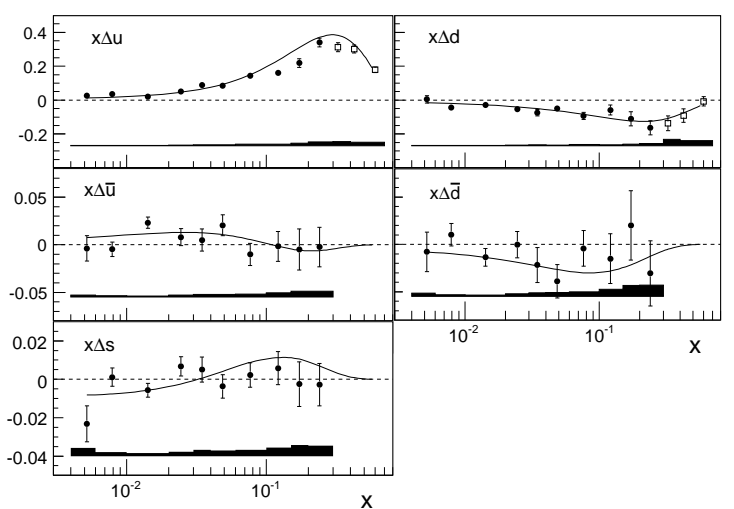

Figure 4: The helicity quark distribution $x \Delta q(x)$ for five flavors compared to the global fit at LO from DSSV [13]. Bands at bottom of graphs represent systematic uncertainties.
The cross-section for semi-inclusive deep-inelastic scattering (SIDIS) in the onephoton exchange approximation contains eight transverse-momentum dependent distribution functions [9]. Some of these can be extracted measuring the azimuthal distribution of the hadrons in the final state [10]. Three distribution functions survive upon integration over the transverse momenta: these are the quark momentum distribution $q(x)$ (where $x$ is the Bjorken variable), the helicity distribution $\Delta q(x)$, and the transversity distribution $\Delta_{T} q(x)$.

The latter is defined as the difference in the number density of quarks with momentum fraction $x$ with their transverse spin parallel to the transversely polarized target and their spin anti-parallel to the target [11]. 


\subsubsection{Semi-inclusive asymmetries and flavor separations}

In parallel to the polarized inclusive DIS measurements, SIDIS events where an additional hadron tags the flavor of the struck quark, were recorded. The data from this reaction $\mu p \rightarrow \mu h X$ are used to extract at LO the helicity quark distributions for each quark flavor separately down to $\mathrm{x}=0.004$. This provides a wider picture of the nucleon spin, however requiring an additional input, the quark Fragmentation Functions (FFs). COMPASS results [12] are shown in Fig. 4 . The curve shows the global QCD fit of DSSV [13] at LO. Sea quark polarized distributions $x \Delta \bar{q}(x)$ and $x \Delta s(x)$ are found to be compatible with zero within the statistical errors. In the future, the SIDIS sector will benefit from more precise determination of quark FFs (Section $€$ ).

\subsection{Collins and Sivers asymmetries in SIDIS}

The existence of an azimuthal asymmetry in transversely polarized leptoproduction of spinless hadrons at leading twist, which depends on a $T$-odd fragmentation function and arises from finalstate interaction effects, was predicted by Collins [14] and is generally known as the Collins effect. In SIDIS the transversity distribution $\Delta_{T} q(x)$ can be measured in combination with the chiral odd Collins fragmentation function $\Delta_{T}^{0} D_{q}^{h}(x)$. The hadron yield $N\left(\Phi_{\text {Coll }}\right)$ can be written as $N\left(\Phi_{\text {Coll }}\right)$ $=N_{0} \times\left(1+f \times P_{t} \times D_{N N} \times A_{\text {Coll }} \times \sin \left(\Phi_{\text {Coll }}\right)\right.$, where $N_{0}$ is the average hadron yield, $f$ the fraction of polarized material in the target, $P_{t}$ the target polarization, $D_{N N}=(1-y) /\left(1-y+y^{2} / 2\right)$ the depolarization factor, $y$ the fractional energy transfer of the muon and $A_{\text {Coll }}$ the Collins asymmetry. The angle $\Phi_{\text {Coll }}$ is the so called Collins angle. It is defined as $\Phi_{\text {Coll }}=\phi_{h}-\phi_{s}$, the difference of the hadron azimuthal angle $\phi_{h}$ and the quark spin azimuthal angle $\phi_{s}$ after the scattering, both with respect to the lepton scattering plane. The measured Collins asymmetry $A_{\text {Coll }}$ can be factorized into a convolution of the transversity distribution $\Delta_{T} q(x)$ and the Collins fragmentation function summed over all quark flavors $q$.

An entirely different mechanism was suggested by Sivers [15] as a possible cause of the transverse spin effects observed in pp scattering. This mechanism could also be responsible for a spin asymmetry in the cross-section of SIDIS of leptons on transversely polarized nucleons. Sivers conjecture was the possible existence of a correlation between the transverse momentum $\vec{k}_{T}$ of an unpolarized quark in a transversely polarized nucleon and the nucleon polarization vector. The correlation between the transverse nucleon spin and the transverse quark momentum is described by the Sivers distribution function $\Delta_{0}^{T} q\left(x, \vec{k}_{T}\right)$. The Sivers effect results in an azimuthal modulation of the produced hadron yield: $N\left(\Phi_{\text {Siv }}\right)=N_{0} \times\left(1+f \times P_{t} \times D_{N N} \times A_{S i v} \times \sin \left(\Phi_{S i v}\right)\right.$. The Sivers angle is defined as $\Phi_{S i v}=\phi_{h}-\phi_{S}$, where $\phi_{S}$ is the azimuthal angle of the target spin vector. The measured Sivers asymmetry $A_{S i v}$ can be factorized into a product of the Sivers distribution function and the unpolarized fragmentation function $D_{q}^{h}(z)$.

\subsubsection{COMPASS measurements}

Since the Collins and Sivers asymmetries are independent azimuthal modulations of the cross section for semi-inclusive deep-inelastic scattering [16], both asymmetries are determined experimentally in a common fit to the same data set, taking into account the acceptance of the spectrometer [17]. In the first plot of Fig. [5 the results for the Collins asymmetry on the proton target are shown as a function of $x$ for positive and negative pions. The asymmetry increases up to about 

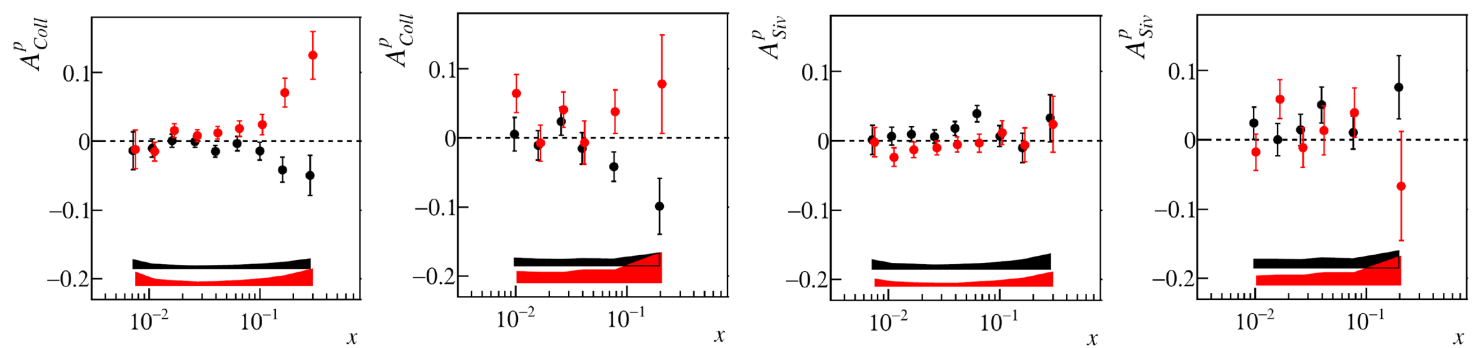

Figure 5: First plot: Collins asymmetry on the proton for positive (black) and negative (red) pions as a function of x. Second one: Collins asymmetry for kaons. Third one: Sivers asymmetry for pions. Last plot: Sivers asymmetry for kaons.

$10 \%$ with opposite sign for negative and positive pions. This result confirms the measurement of a sizable Collins function and transversity distribution. The asymmetry for positive and negative kaons is shown in the second plot of Fig. D. At larger $x$, the asymmetry is different from zero as well and shows opposite signs for positive and negative kaons. The data provide important information for global fits taking into account the Collins fragmentation function from BELLE and the Collins asymmetries from COMPASS and HERMES to obtain constrains to the transversity distribution for $u, d, s$ quarks [18].

In the third plot in the Fig.5 the results for the Sivers asymmetry on the proton are shown as a function of $\mathrm{x}$. The Sivers asymmetry for negative pions is small and statistically compatible with zero. For positive pions the Sivers asymmetry is positive. The Sivers as ymmetry for kaons is shown on the last forth plot. More detailed discussion of the COMPASS results can be find in [19].

\section{Part II: HADRON REACTIONS}

Central production:

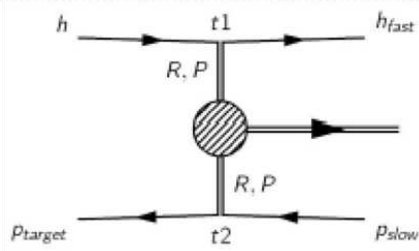

Diffractive dissociation:

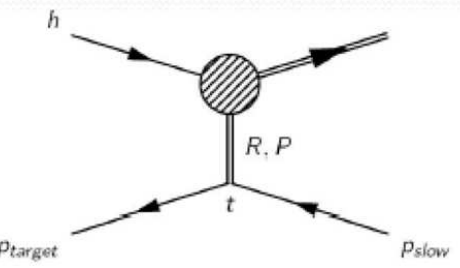

Coulomb production:

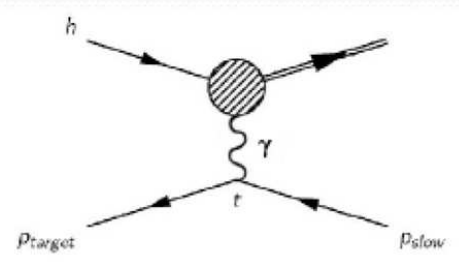

Figure 6: At COMPASS three production mechanisms are accessible. Left: Diffractive dissociation of a beam hadron via Reggeon exchange: forward kinematics; large cross-section $(\sim m b)$; need to separate particles at very small angles; study of $J^{P C}$-exotic mesons. Middle: Central production via double Reggeon fusion: large rapidity gap between scattered beam and produced particles; beam particle looses $\sim 10 \%$ of its energy; particles at large angles; possible source of glueballs. Right: Coulomb production: the pion and kaon polarizabilities measurements.

Forty years after the recognition that quark and gluons are the building blocks of matter, hadronic physics is at a turning point. The quark models of hadrons do not supply a realistic 
picture of the confinement of quarks and gluons in hadrons. A field theoretical based understanding is needed, in the framework of QCD. New theoretical tools have been developed and some experimental data have opened the way, but we are still lacking precise information on two central subjects: the spectroscopy of so-called exotic states, and the spatial structure of the nucleon.

This is the context where, after several years of running with a muon beam to study nucleon spin structure, the COMPASS collaboration in 2008-2009 searched for the exotic states, glueballs or hybrids, through light hadron spectroscopy in high energy pion-proton reactions, using both centrally produced and diffractive events (Fig.6). QCD and derived models predict in particular the existence of $q \bar{q} g$ hybrids, which are difficult to identify experimentally due to mixing with ordinary $q \bar{q}$ mesons. However, some of them might have quantum numbers forbidden for $q \bar{q}$ systems, e. g. $J^{P C}=0^{--}, 0^{+-}, 1^{-+}$. Their observation would therefore provide a fundamental confirmation of QCD. Lattice calculations predict a spin-exotic hybrid with mass within $(1-2) \mathrm{GeV} / c^{2}$ [20]. Some promising candidates have been found for example the $\pi_{1}(1600)$ reported by BNL and VES [21]. A detailed description of the COMPASS results in this field can be find in [22].

\subsection{The COMPASS hadron setup}

For the run with a hadron beam, several major modifications of the COMPASS setup were made. The polarized target was substituted by a liquid hydrogen target with a cell of $40 \mathrm{~cm}$ length and $3.5 \mathrm{~cm}$ diameter, surrounded by a Recoil Proton Detector (RPD) to detect the slow recoil proton produced at large angle in central production and diffractive dissociation. A big effort has been dedicated to minimize the amount of material along the beam path and in the region close to it, thus reducing the background from secondary interactions. New GEM detectors, with a pixels readout in the central region, have been developed [23] and used for a replacement of several of the scintillating fibre detectors tracking the particles at very small angles. A position resolution of 90 $\mu \mathrm{m}$ and timing resolution of $8 \mathrm{~ns}$ were reached.

The negative hadron beam consisted of $96.8 \% \pi^{-}, 2.4 \% K^{-}$and $0.8 \% \bar{p}$ whereas the positive beam consisted of $74.6 \% p, 24.0 \% \pi^{+}$and $1.4 \% K^{+}$, all with momenta of $190 \mathrm{GeV} / \mathrm{c}$. The beam particles were identified by CErenkov Differential counters with Achromatic Ring focus (CEDAR) detectors located upstream of the target [24].

\subsection{Partial Wave Analysis (PWA) and observation of $\pi_{1}(1600)$ exotic state}

First physics result from the COMPASS hadron program were obtained already from the threeday pilot run in 2004. In the analysis, presented in [25], the beam pion was assumed to dissociate diffractively into an intermediate state $X^{-}$. Exchange of a t-channel Pomeron is assumed, for which $X^{-}$gets the same isospin I, G-parity $\mathrm{G}$ and C-parity $\mathrm{C}$ as the incoming pion. $\pi^{-} \mathrm{Pb} \rightarrow \pi^{-} \pi^{-} \pi^{+}$ in COMPASS using a $190 \mathrm{GeV} / \mathrm{c} \pi^{-}$beam provide clean access to meson resonances with masses below $2.5 \mathrm{GeV} / \mathrm{c}^{2}$. The data sample comprised 420000 events. The quantum numbers of $X^{-}$, i.e. the spin J, parity $\mathrm{P}$ and the spin projection M, were disentangled by PWA. The PWA is based on the isobar model and the Zemach formalism. One partial wave is characterized by a set of quantum numbers $J^{P C} M^{\varepsilon}$ [isobar] $\mathrm{L}$, where $J^{P C}$ represents the spin, parity and C-parity of the resonance $\mathrm{X}$, respectively. $\mathrm{M}$ and $\varepsilon$ (reflectivity) describe the spin projection. $\mathrm{X}$ is assumed to decay into a di-pion $[$ isobar $]$ and a bachelor $\pi^{-}$, which have a relative orbital angular momentum $\mathrm{L}$. The isobar 
further decays into a $\pi^{+} \pi^{-}$pair. The PWA is divided into two steps, namely a mass-independent and a mass-dependent fit.

For the subsequent mass-dependent fit a subset of seven waves has been selected: $\quad 0^{-+} 0^{+}\left[f_{0}(980) \pi\right] S, \quad 1^{-+} 1^{+}[\rho \pi] P$ (spin-exotic), $1^{++} 0^{+}[\rho \pi] S, \quad 2^{-+} 0^{+}\left[f_{2} \pi\right] S$, $2^{-+} 0^{+}\left[f_{2} \pi\right] D, 2^{++} 1^{+}[\rho \pi] D$ and $4^{++} 1^{+}[\rho \pi] G$. The intensities and interferences of these waves are parametrized with relativistic Breit-Wigner (BW) and eventually background functions. From this fit, which is shown as red curve overlayed to the mass independent fit results, resonance masses and widths have been obtained.

The Fig.] 7 shows the spin-exotic $1^{-+} 1^{+}[\rho \pi] P$ signal. The mass-dependent fit gives the values of the mass and width of $1660 \pm$ $10_{-64}^{+0}$ and $269 \pm 21_{-64}^{+42} \mathrm{MeV} / \mathrm{c}^{2}$ respectively which is consistent with the hybrid candidate $\pi_{1}(1600)$ [21].

\subsection{Chiral Perturbation Theory (ChPT) predictions test}

The cross section of pion production in $\gamma \pi^{-} \rightarrow \pi^{-} \pi^{+} \pi^{-}$, a subprocess of $\pi^{-} P b \rightarrow$ $\pi^{-} \pi^{+} \pi^{-} P b$, at low momentum transfer $t^{\prime}<$ $0.001(\mathrm{GeV} / \mathrm{c})^{2}$ and a $\pi^{-} \pi^{+} \pi^{-}$invariant mass below the $\rho$ threshold, has been measured using data from 2004 [26]. The results, are in agreement with Leading Order ChPT predictions [27].
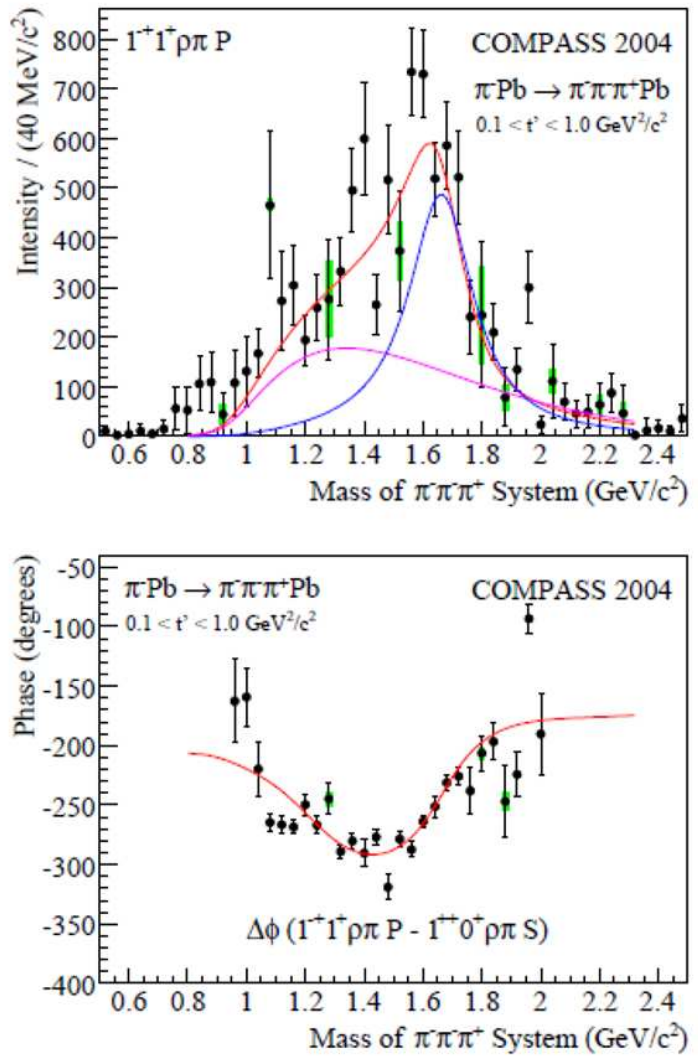

Figure 7: Up: The spin-exotic $1^{-+} 1^{+}[\rho \pi] P$ wave corresponding to the production of the $\pi_{1}(1600)$ hybrid candidate. A background and a BW function have been used in the mass dependent fit to describe this partial wave. Down: Phase differences of the exotic $1^{-+} 1^{+}[\rho \pi] P$ wave to the $1^{++} 0^{+}[\rho \pi] S$ wave. The data points represent the result of the fit in mass bins; the lines are the result of the mass-dependent fit.

\subsection{Search for the $\pi_{1}(1600)$ exotic state in 2008-2009 data}

A preliminary mass independent PWA of $\approx 25 \%$ of the available data from the 2008-09 data set, collected using a hydrogen target and comprising 23 million $\pi^{-} \pi^{+} \pi^{-}$events, confirms the enhancement in the intensity around $M_{X}=1.7 \mathrm{GeV} / \mathrm{c}^{2}$ [28]. The phase motion with respect to the $1^{++}$wave is also consistent with the 2004 data. The $1^{++}$, the exotic $1^{-+}$wave and the phase motion are presented in Fig. 8 (Right). In the Left plot, showing the $1^{-+}$wave, a large bump is observed at around $1.1 \mathrm{GeV} / \mathrm{c}^{2}$ for which the interpretation is under investigation. Mass-dependent fit, leakage studies and background studies of e.g. the Deck effect are ongoing for more definite conclusions. 

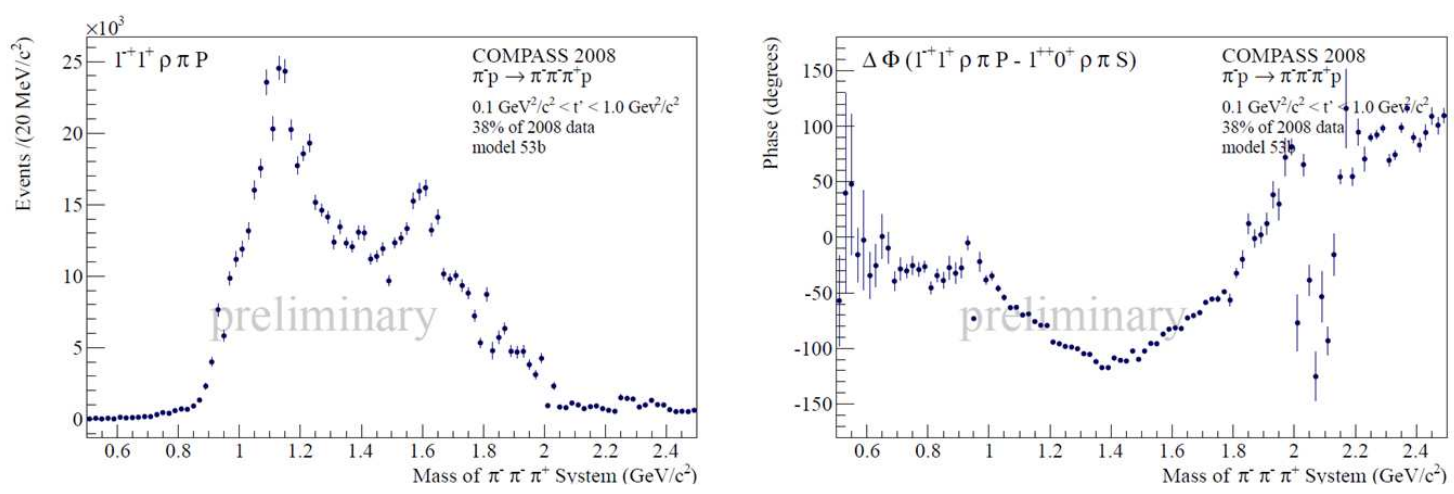

Figure 8: Left: The intensity of the $1^{-+} 1^{+}[\rho \pi] P$ wave. Right: Phase differences of the exotic $1^{-+} 1^{+}[\rho \pi] P$ wave to the $1^{++} 0^{+}[\rho \pi] S$ wave.

\subsection{Channels with $\pi^{0}$}

The two electromagnetic calorimeters allow for studies of neutral final states. One example is the $\pi^{0} \pi^{0} \pi^{-}$final state, which provides an important consistency check with $\pi^{-} \pi^{+} \pi^{-}$channel. Preliminary PWAs, where the $\pi^{-} \pi^{+} \pi^{-}$and the $\pi^{0} \pi^{0} \pi^{-}$final states are compared, show good agreement between the observed wave intensities and the predictions using isospin- and Bose symmetry [29]. Another channel with neutral particles in the final state is the $\eta^{\prime} \pi^{-}$with $\eta^{\prime}$ decaying into $\eta \pi^{+} \pi^{-}$. The first PWA of these data show a strong $1^{-+}$wave, shown in Fig.9 where also the intensity of the $2^{++}$wave and their phase difference are given. However, further studies are needed in order to draw conclusions about the resonance interpretation of the $1^{-+}[30]$. COMPASS can also confirm the decay of $a_{4}(2040)$ into $\eta^{\prime} \pi^{-}$observed by BNL [31].
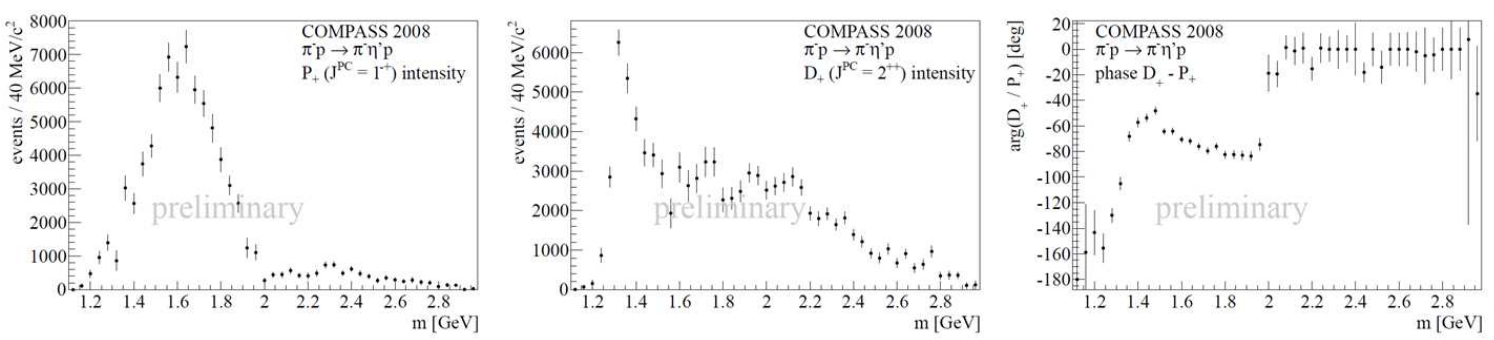

Figure 9: Left: The intensity of the $1^{-+}$, Middle the intensity of the $2^{++}$wave, and Right: the phase difference between $2^{++}$and $1^{-+}$in the $\eta^{\prime} \pi^{-}$channel.

\subsection{Physics with Kaon and proton beams}

The possibility to tag beam kaons with the CEDARs in combination with the RICH identification of final state kaons makes COMPASS an excellent tool for studying kaon diffraction. In a recent study, the reaction $K^{-} p \rightarrow K^{-} \pi^{+} \pi^{-} p_{\text {recoil }}$ is investigated [32]. Recent results from the ongoing PWA show a spectrum of states which is mostly in agreement with previous results from the ACCMOR collaboration [33]. Channels with kaons in the final state are also of interest, in particular $\pi^{-} p \rightarrow(K \bar{K} \pi) \pi^{-} p_{\text {recoil }}$, where COMPASS can provide about an order of magnitude more events than a previous measurement by BNL [34]. 
Data collected with the proton beam have been used to measure the ratio between the cross sections of $p p \rightarrow p p \phi$ and $p p \rightarrow p p \omega$. This provides a test [35] of the Okubo-Iizuka-Zweig (OZI) rule at high energy. The proton beam data are also being used for bary on spectroscopy [36]. Events are selected where the beam proton dissociates diffractively into a baryonic state $X^{+}$which then decays subsequently, via mesonic or baryonic isobars, into the final states $p \pi^{+} \pi^{-}$and $p K^{+} K^{-}$.

\section{Part III: COMPASS II PROPOSAL}

The COMPASS II proposal [37] was approved on December 2010 for an initial data taking of three years. The goal of future studies is to improve the knowledge of the nucleon structure towards a three dimensional picture.

\subsection{DVCS measurement with high energy polarized $\mu^{+}$and $\mu^{-}$beams}

A DVCS measurement with a $160 \mathrm{GeV}$ muon beam was proposed for future COMPASS program [37]. COMPASS will explore the uncharted $x_{B j}$ (up to $\sim 0.27$ ) domain between the HERA collider experiments $\mathrm{H} 1$ and ZEUS and the fixed-target experiments as HERMES and the planned $12 \mathrm{GeV}$ extension of the JLab. In the first phase data will be collected with a liquid hydrogen target to constrain the GPD H. A second phase of data taking with a polarized target (to constrain the GPD E) will be presented in an addendum to the present proposal in the future. Data on DVMP [38] will be recorded simultaneously to the DVCS measurement.

DVCS has the same final state as the competing Bethe-Heitler (BH) process, which is elastic lepton-nucleon scattering with a hard photon emitted by either the incoming or outgoing lepton. The differential cross section for hard exclusive muoproduction of real photons off an unpolarized proton target can be written as ${ }^{2}$

$$
\frac{\mathrm{d}^{4} \sigma(\mu p \rightarrow \mu p \gamma)}{\mathrm{d} x_{B j} \mathrm{~d} Q^{2} \mathrm{~d}|t| \mathrm{d} \phi}=\mathrm{d} \sigma^{B H}+\left(\mathrm{d} \sigma_{\text {unpol }}^{D V C S}+P_{\mu} \mathrm{d} \sigma_{p o l}^{D V C S}\right)+e_{\mu}\left(\operatorname{Re} I+P_{\mu} \operatorname{Im} I\right),
$$

where $P_{\mu}$ is the polarization and $e_{\mu}$ the charge in units of the elementary charge of the polarized muon beam. The interference term $I$ arises due to the interference of the DVCS and the BH processes. The dependence on $\phi$, the azimuthal angle between lepton scattering plane and photon production plane, is a characteristic feature of the cross section. COMPASS offers the advantage to provide various kinematic domains where either BH or DVCS dominate. As the Bethe-Heitler amplitude is well known, the almost pure BH events at small $x_{B j}$ can be used as an excellent reference yield. In contrast the collection of almost pure DVCS events at larger $x_{B j}$ will allow the measurement of the $x_{B j}$-dependence of the $t$-slope of the cross section which is related to the tomographic partonic image of the nucleon. In the intermediate domain, the DVCS contribution will be boosted by the BH process through the interference term. COMPASS is presently the only facility to provide polarized leptons with either charge: polarized $\mu^{+}$and $\mu^{-}$beams. It should be noted that with muon beams one naturally reverses both charge and helicity at once. This feature allows with the same apparatus the measurements of Beam Charge $(C)$ and Spin $(S)$ Difference for Unpo-

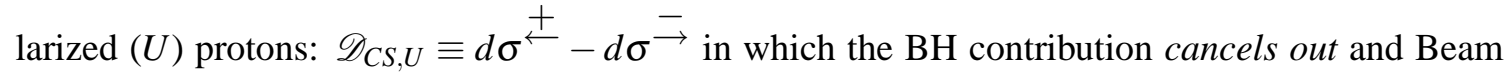

\footnotetext{
${ }^{2}$ For simplicity $\mathrm{d} \sigma$ is used in the following instead of $\frac{\mathrm{d}^{4} \sigma(\mu p \rightarrow \mu p \gamma)}{\mathrm{d} x_{B j} \mathrm{~d} Q^{2} \mathrm{~d}|t| \mathrm{d} \phi}$.
} 

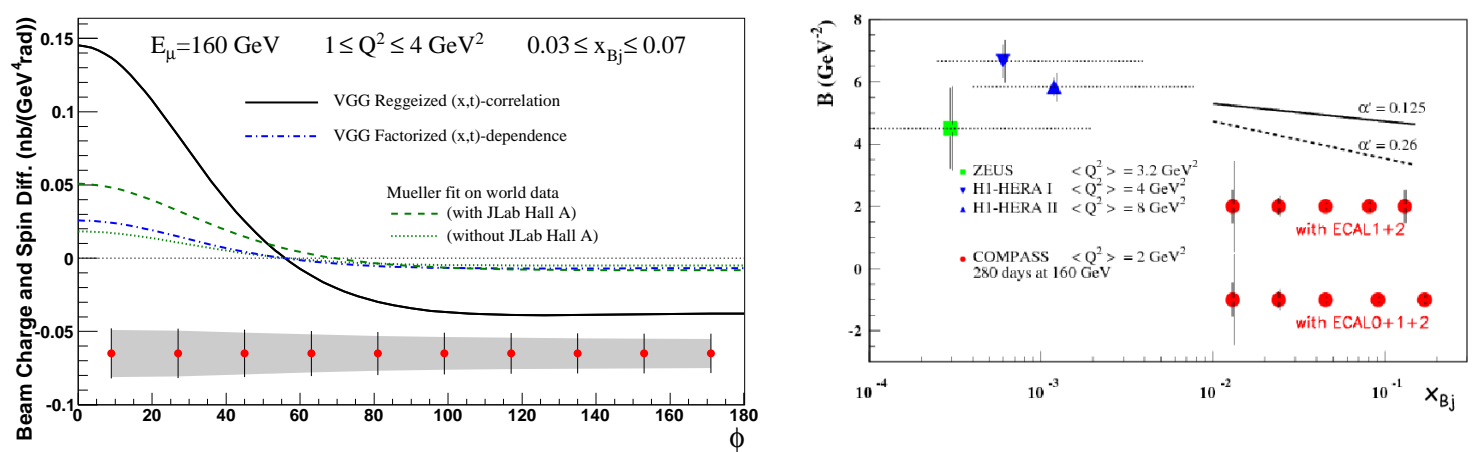

Figure 10: Left: Projected statistical (error bars) and systematic (grey band) accuracy for a measurement of the $\phi$ dependence of the $\mathscr{D}_{C S, U}$ at COMPASS for 2 years of data taking (equivalent to an integrated luminosity of $1222 \mathrm{pb}^{-1}$ ) and an overall global efficiency $\varepsilon_{\text {global }}=0.1$. Right: Projections for measuring the $x_{B j}$ dependence of the $t$-slope parameter $B\left(x_{B j}\right)$ of the DVCS cross section. For comparison some HERA results are shown [39]. The left vertical bar on each data point indicates the statistical error only while the right one includes also the systematic uncertainty, using only ECAL1 and ECAL2 (up) and also ECAL0 (bottom).

Charge and Spin Sum of cross sections: $\mathscr{S}_{C S, U} \equiv d \sigma^{\ddagger}+d \sigma^{\overline{-}}$ in which the BH contribution does not cancel out.

From these measurements the real and imaginary part of Compton Form Factors (CFF) can be extracted. A CFF is a sum over flavors $f$ of convolutions of the respective GPDs with a perturbatively calculable kernel describing the hard $\gamma^{*} q$ interaction. In the difference $\mathscr{D}_{C S, U}$ the analysis of the $\phi$ dependence will provide a measurements of the real part of the corresponding CFF. Fig.10 (Left) shows the projected statistical accuracy in a particular $\left(x_{B j}, Q^{2}\right)$ bin, for a measurement of the $\phi$-dependence of the $\mathscr{D}_{C S, U}$. Fig. 10 (Right) shows the projected statistical accuracy for a measurement at COMPASS of the $x_{B j}$-dependence of the $t$-slope parameter $B\left(x_{B j}\right)$ of the DVCS cross section. Data on $B$ exist only for the HERA collider $x_{B j}$-range from $10^{-4}$ to 0.01 [39]. In the valence region, where no experimental determinations of $B$ exist, some information comes from fits adjusted to form factor data which give $\alpha^{\prime} \simeq 1 \mathrm{GeV}^{2}$ [40]. For the simulation two values $\alpha^{\prime}=0.125$ and $\alpha^{\prime}=0.26$ are shown which correspond to the half and the total of the value for Pomeron exchange in soft scattering processes.

Two DVCS test runs were performed to verify the feasibility of the DVCS program. The 2009 run provided a first direct measurement of the relative contributions of the BH and DVCS events in the COMPASS kinematical region.

Apart of hermetic coverage with electromagnetic calorimetry for future measurements the COMPASS spectrometer has to be upgraded with a $2.5 \mathrm{~m}$ long liquid hydrogen target and a $4 \mathrm{~m}$ long recoil proton detector. A first data taking with the full new set-up is planned for the end of 2012. 


\subsection{SIDIS measurements}

In parallel to the DVCS and DVMP measurements the study of SIDIS will be continued. The additional information provided by a final state hadron allows to access the flavor separated PDFs. These are relatively well constrained in the case of light quarks, but for the strange quark the uncertainties are still considerably large. COMPASS can give valuable input to help constraining the unpolarized strange quark distribution function $s(x)$ in the region of large $\mathrm{x}\left(x_{B j}>0.006\right)$. The final state hadrons from the unpolarized SIDIS processes are identified by the RICH and the calorimeters. From this study the Fragmentation Functions (FF) in general, and the kaon FF in particular will be accessible. Due to the large amount of data, detailed studies of the dependence on several kinematic variables like $x_{B j}, z, Q^{2}$ and $p_{T}$ for hadron multiplicities will be possible, which will also provide high precision inputs to NLO pQCD analyses. A significant improvement of the statistical accuracy of $(s+\bar{s})$ PDF is expected, even with only one week of data-taking.

\subsection{Drell-Yan measurements}
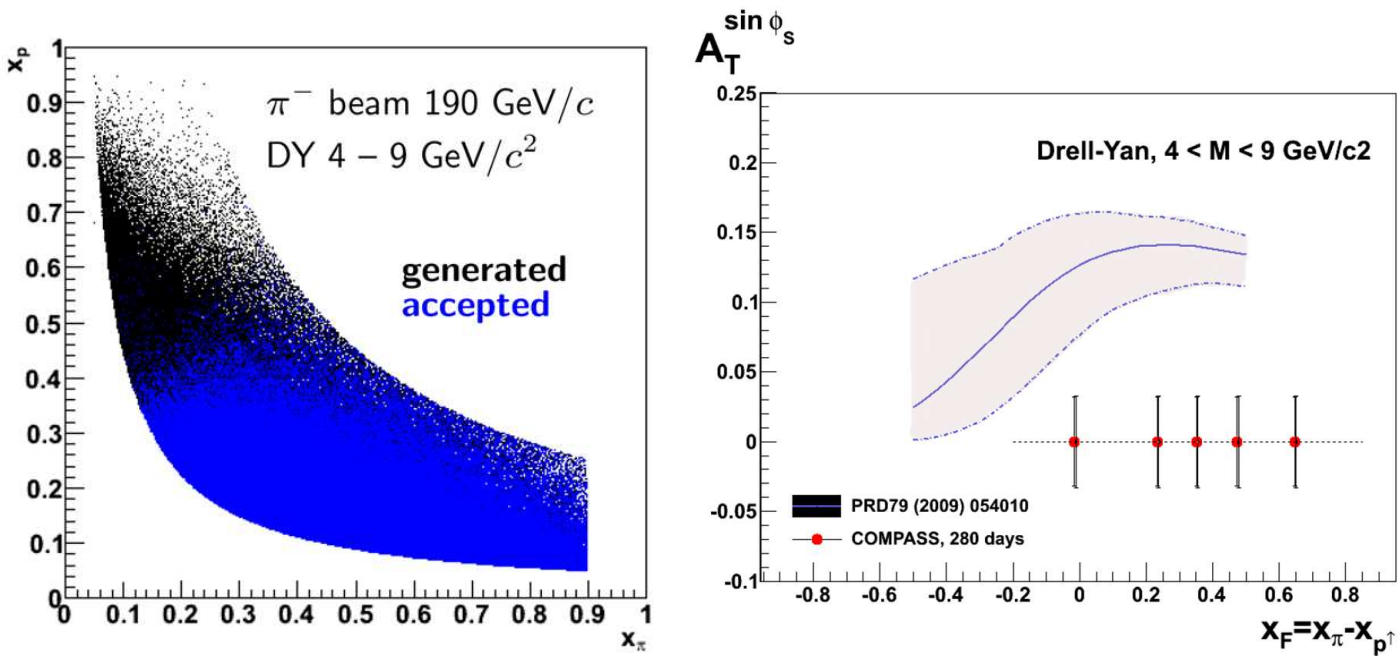

Figure 11: Left: Kinematic range in $x_{\pi}$ vs. $x_{p}$, covered by COMPASS (in blue) for $\mathrm{M}_{\gamma \gamma}>4 \mathrm{GeV} / \mathrm{c}^{2}$ and $\mathrm{M}_{\gamma \gamma}<9 \mathrm{GeV} / \mathrm{c}^{2}$. Right: Statistical accuracy of the Sivers asymmetry in 2 years of data-taking, compared with the theory prediction from [42], for the Drell-Yan COMPASS case in the dimuons high mass region.

Complementary aspects of the nucleon can be studied with TMDs accessed from the polarized Drell-Yan process [41]. The TMDs provide us with a dynamic picture of the nucleon, by taking into account the intrinsic transverse momentum $\vec{k}_{T}$ of its constituents. Until now COMPASS has studied TMDs from SIDIS processes. DY data will allow a test of the factorization ansatz: the sign of the Sivers and Boer-Mulders functions are expected to be opposite in DY and SIDIS. In COMPASS four azimuthal asymmetries can be studied, which are proportional to convolutions of PDFs of the pion and the proton: Boer-Mulders, Sivers, pretzelosity and transversity. The measurement will probe the valence quarks region $x_{p}>0.1$, where the theory predicts sizable asymmetries.

The Drell-Yan measurements will be done with a $\pi^{-}$beam at $190 \mathrm{GeV} / \mathrm{c}$ and a two cells $\mathrm{NH}_{3}$ transversely polarized target. The like-sign muon pairs sample will be used to estimate the combinatorial background, which is expected to be negligible in the high mass region $M_{\gamma \gamma}>4 \mathrm{GeV} / \mathrm{c}^{2}$ 
and $\mathrm{M}_{\gamma \gamma}<9 \mathrm{GeV} / \mathrm{c}^{2}$ where we propose to do the main measurement. Kinematic range of $x_{\pi}$ and $x_{p}$ variables which are the fractions of the momentum carried by the interacting parton in the incoming pion and target proton respectively, covered by COMPASS is shown on Fig.11 (Left). Main part of potentially observed DY events correspond to annihilation of valence quarks $\left(x_{\pi}, x_{p}>0.1\right)$. In 280 days of data-taking, one expects to collect 230000 high mass Drell-Yan events. In Fig.11 (Right) the statistical accuracy of the Sivers asymmetry is shown together with a theory prediction available for the high mass Drell-Yan case at COMPASS, from [42]. Feasibility of Drell-Yan measurements at COMPASS was studied during tests runs. The most important one was performed in 2009. The number of found J $\psi$ 's is found to be in good agreement with the expected yield as well as the number of DY events. COMPASS has the potential to become the first experiment to access the TMDs of the nucleon in a polarized Drell-Yan experiment.

\subsection{Primakoff}

Future COMPASS measurement of pion and kaon polarizabilities will provide a test of ChPT, the theory predicting the strong interaction dynamics of Goldstone bosons. The deviation from the point-like behavior of the pion is revealed by its response in the presence of an electromagnetic field. This effect, called the pion polarizability, is given in lead-
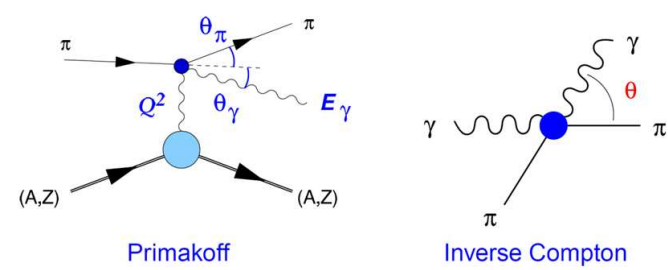

Figure 12: The Primakoff reaction $\mathrm{pA} \rightarrow \mathrm{pA} \gamma$ and embedded in this processus the inverse Compton process $\gamma \pi \rightarrow \gamma \pi$. ing order by a sum of three terms which depend on $\alpha_{s}$ and $\beta_{s}$, the dipole and quadrupole electric and magnetic responses. Although a number of measurements, mainly for $\alpha_{\pi}-\beta_{\pi}$, were performed no firm conclusion yet could be drawn on the comparison to the chiral prediction for $\alpha_{\pi}$ $\beta_{\pi}=(5.70 \pm 1.0) \times 10^{-4} \mathrm{fm}^{3}$ [27].

COMPASS will measure the polarizabilities using the Primakoff reaction, $\mathrm{pA} \rightarrow \mathrm{pA} \gamma$ (Fig.12). Embedded in this reaction is the inverse Compton process $\gamma \pi \rightarrow \gamma \pi$ with the $\gamma$ being deviated at an angle $\theta$. By measuring the modulations in $\theta$ one accesses each of the polarizability terms. With a total measurement time of about 120 days (90 days for $\pi$ and 30 days for $\mu$, planned in 2012) a statistical precision for $\alpha_{\pi}-\beta_{\pi}$ of $0.66 \times 10^{-4} \mathrm{fm}^{3}$ is expected. First measurement of $\alpha_{\pi}+\beta_{\pi}$ is planned also. The statistical precision of $\alpha_{\pi}+\beta_{\pi}$ is expected to be $0.025 \times 10^{-4} \mathrm{fm}^{3}$. The prediction for $\alpha_{\pi}+\beta_{\pi}$ is about $0.16 \times 10^{-4} \mathrm{fm}^{3}$ [27].

The kaon polarizabilities can also be measured in COMPASS for the very first time, thanks to the beam particle identification provided by the CEDAR detectors in the COMPASS beam line.

Reactions with one or two neutral pions instead of a photon in the final state will allow to investigate the chiral anomaly and get further insight into chiral dynamics.

\section{CONCLUSION}

COMPASS is a major player in the study of the nucleon spin structure. Direct measurements of the gluon polarization $\left\langle\frac{\Delta g}{g}\right\rangle$ indicate a small value of the first moment $\Delta G$. The global QCD analysis of $g_{1}$ also shows small values $|\Delta G| \approx 0.2-0.3$. To solve the "spin crisis" 10 times bigger 
$|\Delta G|$ is needed. From another side these results are the probable signature for a predominant role of the angular orbital momentum of quarks and gluons in the nucleon spin decomposition.

In the "longitudinal" spin physics the helicity quark distributions $x \Delta q(x)$ were measured, in the"transverse" spin physics the evidence that transversity PDF and Sivers function are different from zero was obtained. The study of transverse spin effects needs further precise measurements and the COMPASS facility is the only place where SIDIS can be measured at high energy.

COMPASS has excellent potential to contribute for searching QCD allowed states like multiquarks, glueballs and hybrids because it has access to 3 production mechanisms: diffractive dissociation, central and coulomb productions. A large amount of data, 10-100 times world statistics, were collected with hadron beam in 2008-2009. A candidate for $\pi_{1}(1600)$ exotic state in the 2004 short pilot run was already observed. Preliminary analysis of 2008-2009 data confirms this observation.

COMPASS has also a great potential in such new fields as DVCS for the study of GPDs, polarised Drell-Yan to study TMDs and in the measurements of pion (kaon) polarisabilities.

The work has started to get the spectrometer upgraded for the new program and to be ready for data taking in 2012, 2014-2016.

\section{References}

[1] P. Abbon et al.,Nucl.Instr. Meth. A577 (2007) 455.

[2] J. Ashman et al., Phys. Lett. B206 (1988) 364.

[3] J. Ellis and R.L.Jaffe, Phys. Rev. D9 (1974) 444, Phys. Rev. D10 (1974) 1669.

[4] C. Franco, International Workshop on Hadron Structure and Spectroscopy 2011, Paris. 4-6 April 2011.

[5] E.S. Ageev et al., Phys. Lett. B633 (2006) 25.

[6] B. Adeva et al., Phys. Rev. B70 (2004) 012002.

[7] A. Airapetian et al., Phys. Rev. Lett. 84 (2000) 2584.

[8] E. Leader AIP Conf.Proc.1149 (2009) 381-384.

[9] P.J. Mulders, R.D. Tangerman, Nucl. Phys. 461,(1997) 197.

[10] J.C. Collins et al., Nucl. Phys. B420,(1994) 565.

[11] X. Artru and J.C. Collins, Z. Phys. C69,(1996) 277.

[12] M. Alekseev et al., COMPASS Phys. Lett. B 693 (2010) 227.

[13] D. de Florian, R. Sassot and M. Stratmann (DSS) Phys. Rev. D 75 (2007) 114010; D. de Florian, R. Sassot, M. Stratmann and W. Vogelsang (DSSV), Phys. Rev. Lett. 101 (2008) 072001.

[14] J. Collins, Nucl. Phys. B 396 (1993) 161..

[15] D. Sivers, Phys. Rev. D 41 (1990) 83.

[16] D. Boer and P.J. Mulders, Phys. Rev. D57, (1998) 5780

[17] V.Yu. Alexakhin et al., Phys. Rev. Lett. 94,(2005) 202002; E.S. Ageev et al., Nucl. Phys. B765, (2007) 31; M.G. Alekseev et al., Phys. Lett. B692 (2010), 240. and M. Alekseev et al., Eur. Phys. J. C64 (2009), 171. 
[18] A. Bacchetta and M. Radici, Phys. Rev. D67, 094002 (2003), Phys. Rev. D69, 074026 (2004) and Phys. Rev. D74, 114007 (2006).

[19] Ch. Schill, XIX Int'1 Workshop on Deep-Inelastic Scattering, Newport New, USA, April 10-15, 2011.

[20] F.E. Close and P.R. Page Nucl. Phys. B 443 (1995) 233.

[21] G.M. Beladidze et al., Phys. Lett. B 131 (1993) 276; G.S. Adams et al., Phys. Rev. Lett. 81 (1998) 5760; Y. Khoklov et al. Nucl. Phys. A 663(2000) 596; E.I. Ivanov et al. Phys. Rev. Lett. 86 (2001) 3977; J. Kuhn et al., Phys. Lett. B 595(200) 109; D.V. Amelin et al., Phys. Atom. Nucl. 68 (2005) 359; M. Lu et al., Phys. Rev. Lett. 94 (2005) 032002.

[22] K. Schöning, Rutherford Centennial Conference on Nuclear Physics, Manchester, UK, August 8-12, 2011.

[23] T. Nagel, Proc. of the 10th ICATPP Conference, Como, Italy (2007).

[24] C. Bovet et al. 1982 The CEDAR counters for particle identification in the SPS secondary beams: a description and an operation manual CERN report SPS-82-13.

[25] M. Alekseev at al., Phys. Rev. Lett. 104 (2010) 241803.

[26] M.G. Alekseev at all CERN-PH-EP 2011-176 Subm. PRL

[27] N. Kaiser and J.M.Friedrich Eur. Phys. J. A 36 (2008) 181.

[28] F. Haas, Proceedings from the XIV International Conference on Hadron Spectroscopy (Hadron 2011), Munich, Germany, 2011 arXiv:1109.1789.

[29] F. Nerling, ibid., arXiv:1108.5969.

[30] T. Schlüter, ibid., arXiv:1108.6191

[31] E.I. Ivanov et al. Phys. Rev. Lett. 86 (2001) 3977.

[32] P. Jasinski, Proceedings from the XIV International Conference on Hadron Spectroscopy (Hadron2011), Munich, Germany, arXiv:1109.1789.

[33] C. Daum et al. Nucl. Phys. B 187 (1981) 1.

[34] J.H. Lee et al., Phys. Lett. B 323 (1994) 327.

[35] J. Bernhard Rutherford Centennial Conference on Nuclear Physics, Manchester, UK, August 8-12, 2011.

[36] A. Austregesilo, Proceedings from the XIV International Conference on Hadron Spectroscopy (Hadron 2011), Munich, Germany, 2011 arXiv:1109.0230

[37] COMPASS-II proposal, SPSC-P-340.

[38] D. Mueller et al., Fortsch. Phys. 42 (1994) 101; A.V. Radyushkin, Phys. Lett. B 385 (1996) 333; Phys. Rev. D 56 (1997) 5524.

[39] H1, A. Aktas et al., Eur. Phys. J.C 44 (2005) 1; F.D. Aaron et al., Phys. Lett. B 659 (2008) 796, ZEUS, S. Chekanov et al., DESY-08-178, arXiv:hep-exp:0812.2517v3.

[40] M. Diehl et al., Eur. Phys. J. C 39 (2005) 1, M. Guidal et al., Phys. Rev. D 72 (2005) 054013.

[41] S. Arnold et al., Phys. Rev. D 79 (2009) 034005.

[42] M. Anselmino et al, Phys. ReV. D 79 (2009) 054010. 